\title{
Silver-Russell Syndrome-like Features in a Patient Carrying a Novel NF1 Mutation
}

\author{
KATHARINA WIMMER, MATHIAS DECKER, ERTAN MAYATEPEK, HARALD BEIGLBÖCK, \\ THOMAS EGGERMANN, HILDEGARD KEHRER-SAWATZKI, \\ CHRISTA FONATSCH, AND THORSTEN ROSENBAUM \\ Department of Human Genetics [K.W., H.B., C.F.], Clinical Institute of Medical and Chemical Laboratory \\ Diagnostics, Medical University of Vienna, A-1090 Vienna, Austria; Department of General Pediatrics \\ [M.D., E.M., T.R.], Heinrich-Heine-University Duesseldorf, D-40225 Duesseldorf, Germany; Institute of \\ Human Genetics [T.E.], RWTH Aachen, D-52074 Aachen, Germany; Department of Human Genetics \\ [H.K.-S.], University of Ulm, D-89081 Ulm, Germany
} \begin{abstract}
\begin{tabular}{|l|} 
MBS \\
Mutations in the NF1 gene (17q11.2) cause neurofibromatosis \\
type 1 (NF1), a pleiotropic and progressive autosomal dominant
\end{tabular} disorder with marked variability of clinical expression. Clinical diagnosis is usually readily achieved in most adult and adolescent patients due to the presence of at least two of the classic signs of NF1. However, the absence of many of the diseasedefining features in young children frequently renders definite diagnosis impossible in this age group. Particularly, clinical diagnosis is challenging in young patients whose phenotypical presentation does not lie within the common spectrum of "typical" NF1 features. Sensitive and reliable molecular genetic testing can be of great help in these cases. Here, we report clinical and molecular findings in a 2-year-old boy with features of NF1.
\end{abstract}

Severe growth retardation together with other dysmorphic features was also suggestive for Silver-Russell syndrome (SRS) in this patient. Molecular genetic testing identified a novel NF1 mutation and, thus, enabled a confident NF1 diagnosis despite the unusual phenotypical presentation in this patient. (Pediatr Res 58: 1265-1268, 2005)
CLS, café-au-lait spots
Abbreviations
CSH1, somatomammotropin hormone 1
MRI, magnetic resonance imaging
NF1, neurofibromatosis type 1
SRS, Silver-Russell syndrome

NF1 (MIM 162200) is one of the most common autosomal dominant disorders, affecting approximately one in 3500 individuals. A hallmark of NF1 is the extreme heterogeneity in clinical expression $(1,2)$. Each of the classic signs of NF1 such as café-au-lait spots (CLS), iris Lisch nodules, and cutaneous neurofibromas are found in more than $90 \%$ of the patients. Other features are found less frequently. In general, the major clinical features appear in an age-dependent order. Multiple CLS are usually the first clinical sign in NF1. However, CLS are not exclusively found in NF1 but can also be a feature of other conditions including SRS.

SRS was originally described by Silver et al. in 1953 (3) followed by the description of five additional children by Russell et al. in 1954 (4). Affected children are characterized by severe retardation of length and weight at birth, short stature

Received January 3, 2005; accepted March 23, 2005

Correspondence: Thorsten Rosenbaum, M.D., Department of General Pediatrics, Heinrich-Heine-University Duesseldorf, Moorenstr. 5, D-40225 Duesseldorf, Germany: e-mail: rosenbaum@med.uni-duesseldorf.de.

Supported by Hochschuljubiläumsstiftung der Stadt Vienna project no. H1029/2004.

DOI: 10.1203/01.pdr.0000183661.81772.f8 in later life, and typical craniofacial abnormalities with small, triangular face and prominent forehead resulting in a pseudohydrocephalic appearance (5). In addition, there is a wide variety of less constant findings including skeletal asymmetry, fifth finger clinodactyly, psychomotor retardation, and CLS (6). This phenotypical variance might reflect the heterogeneous etiology of SRS. While most cases seem to be sporadic, various modes of inheritance and involvement of several chromosomes have been described (7). Most consistently, maternal uniparental disomy of chromosome 7 (matUPD7) is observed in about $10 \%$ of patients with a clinical diagnosis of SRS. Another candidate region is localized on distal $17 \mathrm{q}$ within the growth hormone gene cluster.

In this report, we present a patient with multiple CLS, severe growth retardation, and various dysmorphic features. Although NF1 appeared to be the most likely cause for this phenotype, the patient did not fulfill the diagnostic criteria as proposed by the National Institutes of Health consensus conference on neurofibromatosis in 1988 (8). Therefore, a clinical diagnosis of NF1 could not be established in a strict sense. In contrast, almost all clinical features of this patient were within the phenotypical spectrum of SRS. Therefore, we performed mo- 
lecular genetic analysis for SRS and NF1 in the patient. The results of the testing and the clinical features of the patient are discussed in this report.

\section{METHODS}

Case. The boy presented here was born as the sixth child of a German family; all other children in the family were boys as well, and none of them was reported to have any severe illnesses, developmental retardation, or suspicious phenotypical features. His parents were healthy, body measurements (height, weight, head circumference) were normal, and there was no history of CLS or any other NF-associated features. After an uneventful pregnancy, he was delivered at 34 weeks of gestation by cesarean section due to a breech presentation. Body weight at birth was $2270 \mathrm{~g}$ (50th percentile), height was $48 \mathrm{~cm}$ (75th percentile), and head circumference was $33.5 \mathrm{~cm}$ (50th-75th percentiles). Apgar scores ( 1 and 5 minutes postpartum) were 8 and 9 , respectively. The first clinical examination of the child showed bilaterally undescended testes but no other pathologic finding. Recurrent failure to feed well during the newborn and infant period prompted laboratory tests of thyroid function and revealed slight hypothyroidism. However, therapy with sodiumL-thyroxine, which is maintained date, was unable to improve the feeding difficulties. At the age of 10 months, CLS appeared and suggested NF1 as underlying disease. The boy was then presented at the NF1 clinic of the local children's hospital for further evaluation. Except for CLS (Fig. 1),examination of the boy did not reveal any other NF1-associated finding. Similarly, eye examination was entirely normal. MRI of the brain showed a small hyperintense focal lesion in the region of the left caudate nucleus. This is a typical finding in pediatric NF1 patients and thus corroborating the provisional diagnosis of NF1, although the diagnostic criteria as defined by the National Institutes of Health consensus conference were not (yet) fulfilled in the patient.

While CLS did not significantly change over time, feeding problems and delay in psychomotor development appeared to deteriorate in the following months. In addition, the mother had noted inadequate heavy sweating of the boy. At the age of 2 years, the boy was first presented in our NF clinic. At that time, the boy had just acquired the ability to walk alone but was still unable to speak. The following investigations were approved by our review board for good clinical practice and were performed after informed consent had been obtained from the boy's parents. Neurologic examination revealed generalized muscular hypotonia and absent tendon reflexes. His height $(77 \mathrm{~cm})$ was $5 \mathrm{~cm}$ below the third percentile, his weight $(7855 \mathrm{~g})$ was $2500 \mathrm{~g}$ below the third percentile. Only the head circumference $(48.3 \mathrm{~cm})$ was within normal range (10th-25th percentiles). Together with low-set ears and prominent forehead, this resulted in a pseudohydrocephalic appearance (Fig. 1). The anterior fontanel was wide open but not bulging. Physical examination of the heart, lungs, and abdomen was normal. Both testes were undescended with otherwise normal male genitalia. Scattered over the whole body there were about 20 CLS, several of them were more than $5 \mathrm{~mm}$ in diameter. Axillary or inguinal freckling and (sub)cutaneous neurofibromas were not noted. There were no osseous malformations except for bilateral fifth finger clinodactyly (Fig. 1).
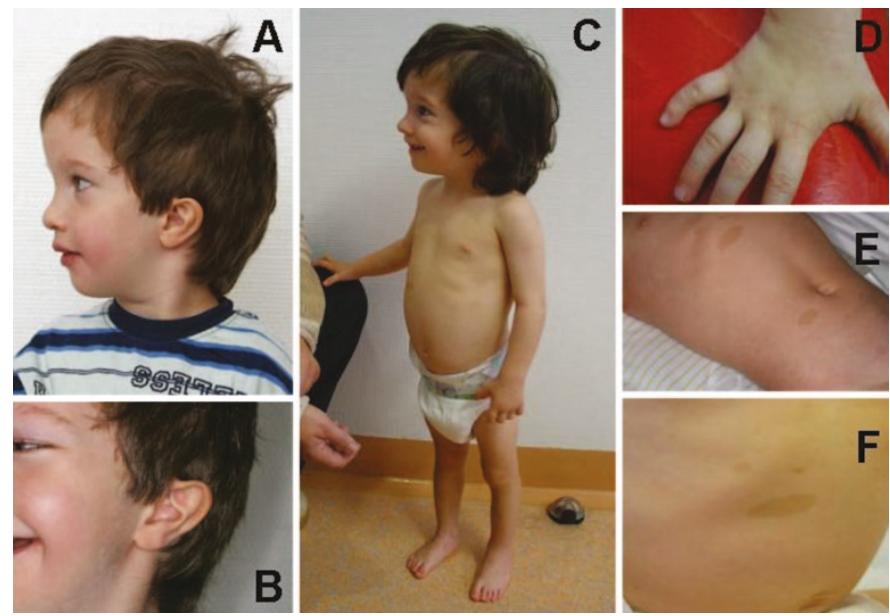

Figure 1. Clinical photographs of the reported patient at the age of $2(C, D$, $F)$ and 4 years $(A, B, E$,). Cutaneous CLS $(E, F)$ suggested NF1 as underlying disease but were also compatible with the diagnosis of SRS. Short stature $(C)$, fifth finger clinodactyly $(D)$, and the pseudohydrocephalic appearance with prominent forehead $(A, C)$ and low-set ears $(A, B)$ were rather indicative of SRS.
Laboratory investigations showed normal hematologic and coagulation parameters as well as normalized thyroid function with medication with sodium-L-thyroxine. Since a radiographic examination of the left hand showed a significantly delayed skeletal maturation (bone age 14 months, chronologic age 24 months), additional laboratory investigations were done to check for growth hormone $(\mathrm{GH})$ deficiency. However, serum levels for IGF-1 (83 $\mathrm{ng} / \mathrm{mL} ; 50$ th-95th percentiles) and GH-dependent IGF-binding protein 1 (IGFBP3, $3.2 \mu \mathrm{g} / \mathrm{mL}$; >95th percentile) did not suggest GH deficiency so that measurement of $\mathrm{GH}$ in response to stimulation was postponed.

The boy again presented at the age of 3 years 5 months. There was clear developmental progress despite significant speech delay and failure to thrive. His height $(88.2 \mathrm{~cm})$ was still $7 \mathrm{~cm}$ below the third percentile, his weight $(11.5$ $\mathrm{kg}$ ) was $1 \mathrm{~kg}$ below the third percentile. Head circumference $(50.8 \mathrm{~cm})$ was at the 50th percentile. Delay of skeletal maturation was even more severe with a bone age of 17 months (Fig. 2).the phenotypical appearance of the boy had remained unchanged. A control MRI of the brain was performed that again showed the focal hyperintense lesion in the left caudate nucleus. In addition, similar lesions could be demonstrated in the cerebellum and the pontine region without mass effect or focal contrast enhancement.

Molecular genetic and molecular cytogenetic analysis. To test for uniparental disomy of chromosome 7 (UPD7) and loss of the somatomammotropin hormone 1 (CSH1), microsatellite markers from the long and the short arm of chromosome 7, i.e. D7S493, D7S481, D7S636, and D7S2446, as well as from the GH gene cluster containing CSH1 on chromosome 17, D17S254, were genotyped. DNA was extracted from peripheral blood lymphocytes of the patient and his mother and polymerase chain reaction (PCR)-amplified using fluorescence-labeled primers according to standard protocols. PCR products were separated and analyzed on an automated sequencer (ABI377, Applera, Darmstadt, Germany).

For mutation analysis in the $N F 1$ gene, first fluorescent in situ hybridization (FISH) analysis was performed with NF1 gene-specific probes RP11-142O6, CITB-23O13, and RP11-805L22, previously described by Jenne et al. (9). Second, protein-truncation testing (PTT) was used to find small sequence alterations in the NF1 gene. Briefly, total RNA of the patient and of healthy control individuals was extracted from phytohemagglutinin (PHA)-stimulated short-term lymphocyte cultures treated with puromycin (10). The entire cDNA of the NF1 gene was reverse-transcriptase-PCR (RT-PCR) amplified in five overlapping fragments using primer pairs as described (11). Subsequently, PCR products were subjected to a transcription/translation reaction and the

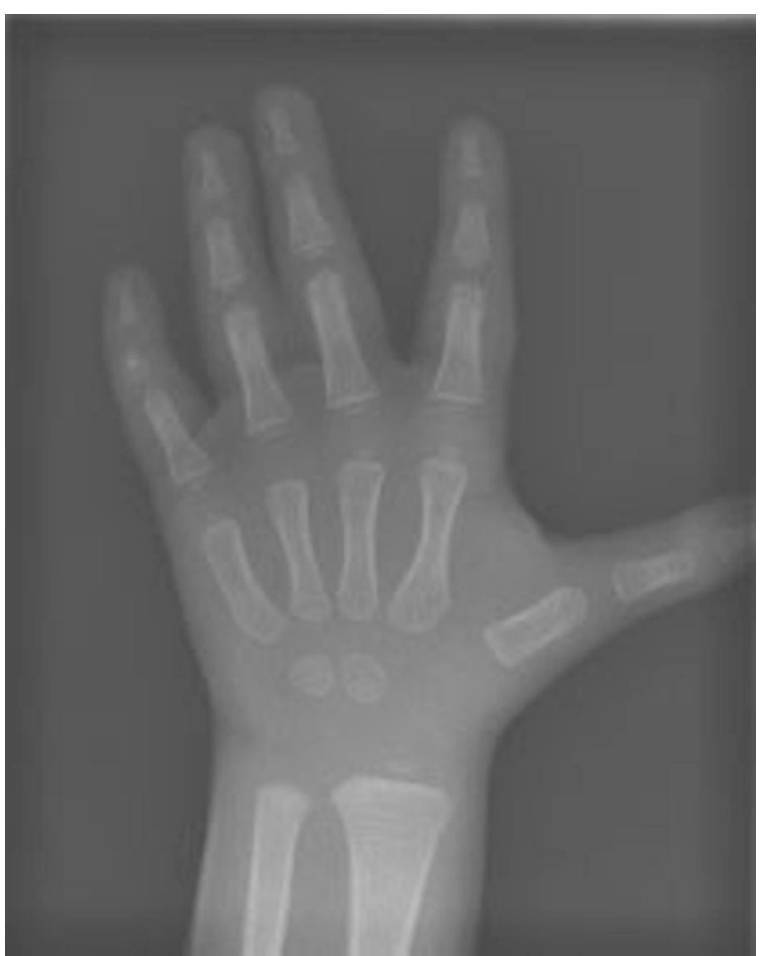

Figure 2. Radiograph of the patient's left hand. Brachymesophalangism of the fifth finger with prominent hypoplasia of the middle phalanx. Otherwise normal anatomy with normal bone density. Chronologic age 3 years 5 months, average skeletal age 1 year 5 months with dissociation (radius/ulna: 1 year 5 months; carpus 1 year, metacarpalia 1 year 5 months, phalanges 1 year 7 months). 
synthesized polypeptide fragments were separated on a SDS-polyacrylamide gel. A primer located in exon 11 (5'-TCATGAAGAACCAGCAGAGC-3') was used for sequencing of the RT-PCR product of the fragment S1. PCR amplification of exon 10A was performed with the primer pair NF1ex10aF (5'-GGAAATCATGGTGTGTGTTTGC- $\left.3^{\prime}\right)$ and NF1ex10aR (5'-CAACATACCACAACATTTAGAGG-5'). The resulting PCR product was sequenced using an automated sequencer (LiCor).

\section{RESULTS}

Maternal uniparental disomy of chromosome 7 (UPD 7) has been described in approximately $10 \%$ of patients with SRS phenotype [reviewed in Ref. 7)]. Furthermore, several SRS patients with paternally inherited heterozygous deletion of the chorionic $\mathrm{CSH} 1$ gene have been described (12). Therefore, four highly polymorphic microsatellites from chromosome 7 , as well as a microsatellite marker located in the $\mathrm{GH}$ gene cluster were genotyped in the patient and his mother. The results did not reveal evidence of UPD 7 nor a deletion of the GH gene cluster region in the patient.

The molecular genetic and molecular cytogenetic analysis of the NFl gene was performed in two steps. An initial FISH analysis did not reveal indication for a microdeletion or other rearrangements affecting the NF1 locus. For further mutation analysis of the NF1 gene, PTT was used. The analysis of the first segment (S1) nucleotides c.1-1868 (exon 1 to 12A) of the NF1 cDNA revealed in the patient a shortened fragment in addition to the normal polypeptide product (Fig. $3 A$ ).fore, the RT-PCR product of S1 was subjected to sequencing analysis and by this means a T to A transversion at nucleotide 1299 of the coding sequence in exon 10A was identified. The nucleotide position is calculated from the initiation codon in NFI mRNA NM_000267.1, where position +1 corresponds to the A of the ATG, which is located at nucleotide 212. To our knowledge, this mutation (c.1299T >A) has so far not been described. The transversion creates a premature termination codon at amino acid position 433 (p.Tyr433×) and was confirmed also at the genomic level by sequencing exon $10 \mathrm{~A}$ of the patient (Fig. 3B).

\section{DISCUSSION}

NF1 usually is a straightforward diagnosis in adolescents and adults presenting with typical features of the disease. In
A

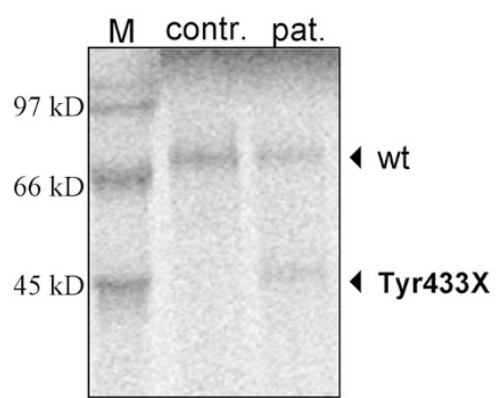

B

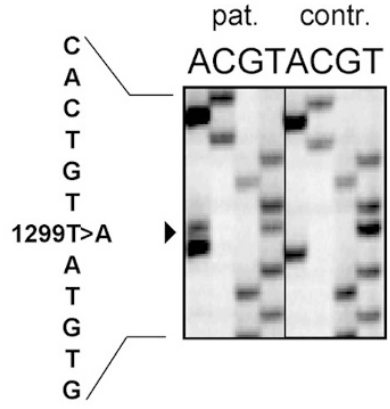

Figure 3. (A) PTT results of segment S1 of a control person (C) and our patient $(\mathrm{P})$. The wild-type (wt) and aberrant band caused by a premature termination codon p.Tyr $433 \times$ are indicated. (B) Sequencing results of exon $10 \mathrm{~A}$ and its adjacent intronic sequences of the patient and a control individual (contr.). In the patient, heterozygosity for A and T at the nucleotide 1299 of the coding sequence is indicated by an arrowhead. infants and young children, however, correct clinical diagnosis might be difficult and can be especially challenging when the phenotypical presentation does not lie within the common spectrum of typical NF1 features. This is exemplified by the patient described here whose phenotype was compatible with the diagnosis of NF1 but who also showed a significant overlap with another genetic condition, SRS.

The diagnostic criteria for NF1 initially established in 1988 by the National Institutes of Health consensus conference were written stringently to avoid misclassification of subjects included in studies aimed at the identification of the NFI and neurofibromatosis type 2 (NF2) genes (8) consensus development conference. These clinical criteria allow unambiguous diagnosis in 94\% of patients by the age of 6 years (13). However, younger children usually present with multiple CLS as the sole criterion, which is not sufficient to establish a correct diagnosis in the absence of a positive family history. Although NF1 is by far the most likely diagnosis in these children, CLS are not pathognomonic for NF1 and may be indicative of various other conditions (14). In this situation, it might be helpful to look for other NF1-associated features that are not included in the National Institutes of Health diagnostic criteria list but may confirm the provisional diagnosis NF1 as an underlying disease. Cranial MRI abnormalities such as hyperintense lesions on T2-weighted images usually appear early in childhood and are found in up to $87 \%$ of all NF1 children (15). However, these unidentified bright objects also present in our patient are not recommended to be used as a diagnostic criterion since the data on their specificity for NF1 are scarce (16).

Other prominent features of the patient presented here were poor weight gain, reduced growth, and relative macrocephaly. Although many reports have described short stature and macrocephaly as a consistent finding in NF1, these growth abnormalities usually become apparent in later childhood and adolescence $(17,18)$. In contrast, our patient exhibited failure to thrive and reduced growth rate throughout infancy and early childhood. This abnormal growth pattern and several other phenotypical features (Table 1)as underlying disease but were rather suggestive of SRS, a condition including multiple CLS in the syndromal expression pattern. However, the fact that our patient showed normal intrauterine growth was not in line with this differential diagnosis since most SRS patients show a severe reduction of weight and length at birth (6). Thus, clinical examination alone did not allow a clear diagnosis in this patient since he exhibited typical features of both NF1 and SRS.

We therefore took a molecular genetic approach and were able to identify, to the best of our knowledge, a novel nonsense mutation of the NF1 gene (c.1299T >A, but we could not provide molecular evidence of SRS. Since the genetic basis of SRS is not yet fully understood and molecular analysis yields a positive result in only about $10 \%$ of SRS patients, this result does not exclude mutations in other genes. Despite these limitations, it is highly likely that NF1 is the underlying disease exhibiting an unusual phenotypical expression including SRSlike features in our patient.

Reviewing more recent studies, it appears that no final concordant conclusion has been found for the question of whether the growth profiles of NF1 patients reveal that to some 
Table 1. Phenotypical features of the reported patient compatible with the clinical diagnosis of SRS, NF1, or both

\begin{tabular}{lll}
\hline \multicolumn{1}{c}{ SRS } & \multicolumn{1}{c}{ SRS and NF1 } & \multicolumn{1}{c}{ NF1 } \\
\hline $\begin{array}{l}\text { Relative macrocephaly with } \\
\text { low-set ears and prominent }\end{array}$ & CLS & MRI hyperintensities \\
forehead & & \\
Feeding problems & Short stature & Muscular hypotonia \\
Retarded bone age & Motor delay & \\
$\begin{array}{l}\text { Delayed closure of anterior } \\
\text { fontanel }\end{array}$ & Speech delay & \\
Clinodactyly V & & \\
Excessive sweating & & \\
Cryptorchism & & \\
\hline
\end{tabular}

extent stature is reduced in all NF1 patients (19) or whether stunted height is likely to affect only a portion of NF1 patients (18). The latter study indicated that there are no prominent differences in height between NF1 and normal subjects up to 7 (girls) and 12 (boys) years old, respectively. Thereafter, height velocity and relative height (standard deviation scores or percentiles) decrease with respect to healthy peers, reaching a mean adult height close to the 25th percentile for the general population. Moreover, the percentage of patients with true short stature (lower than the third percentile) increases from childhood (5\%) to late puberty (18\%), and final height is significantly below the genetic target and predicted adult height calculated just before or at the beginning of puberty (20). These clinical data suggest that alterations of stature in NF1 are the result of lower growth velocity during adolescence.

The molecular mechanisms by which NFI haploinsufficiency leads to this growth pattern are unknown. In addition, it is unclear whether a specific type of NF1 mutations and/or other genetic factors (modifiers) are responsible for altered growth behavior. To our knowledge, a similar course as in our patient has only been described in a single NF1 patient with Noonan syndrome features including ptosis, epicanthic folds, low posterior hairline, low-set ears, and pulmonary stenosis [case 5 of Baralle et al. (21)]. In contrast to our patient who harbors a truncating mutation in exon 10A, an in-frame 3-bp deletion in exon 25 was found in the patient described by Baralle et al. Recently, Spiegel et al. (personal communication) found that patients with the common NF1 microdeletion encompassing a 1.5-Mb DNA segment show overgrowth during childhood. Thus, there is accumulating evidence that genetically different subgroups of NF1 patients show a growth pattern that differs not only from the normal population but also most NF1 patients.

The case described here exemplifies the difficulties of diagnosing NF1 in infants and young children with a rare phenotypical presentation. Even 14 years after cloning the gene (22-24), molecular genetic NF1 diagnostics is still considered a major challenge. However, in recent years, approaches have been developed that reach mutation detection rates of up to 95\% (25). It appears to be essential for the success of these approaches that they combine an RNA-based assay, such as that here applied, and improved PTT, with additional supplemental methods to identify missense mutations as well as whole gene and multiexon deletions. As presented here, these powerful mutation detection technologies do not only enable a confident NF1 diagnosis in patients for whom symptomatic diagnosis is not always straightforward but will also enhance our understanding of possible pathogenetic mechanisms underlying rare phenotypical presentations of NF1.

Acknowledgments. We thank the patient and the family for their cooperation.

\section{REFERENCES}

1. Easton DF, Ponder MA, Huson SM, Ponder BA 1993 An analysis of variation in expression of neurofibromatosis (NF) type 1 (NF1): evidence for modifying genes. Am J Hum Genet 53:305-313

2. Tonsgard JH, Yelavarthi KK, Cushner S, Short MP, Lindgren V 1997 Do NF1 gene deletions result in a characteristic phenotype? Am J Med Genet 73:80-86

3. Silver HK, Kiyasu W, George J, Deamer WC 1953 Syndrome of congenital hemihypertrophy, shortness of stature and elevated urinary gonadotropins. Pediatrics 12:368-376.

4. Russell A 1954 A syndrome of intra-uterine dwarfism recognizable at birth with cranio-facial dysostosis, disproportionately short arms, and other anomalies (5 examples). Proc R Soc Med 47:1040-1044

5. Price SM, Stanhope R, Garrett C, Preece MA, Trembath RC 1999 The spectrum of Silver-Russell syndrome: a clinical and molecular genetic study and new diagnostic criteria. J Med Genet 36:837-842

6. Wollmann HA, Kirchner T, Enders H, Preece MA, Ranke MB 1995 Growth and symptoms in Silver-Russell syndrome: review on the basis of 386 patients. Eur J Pediatr 154:958-968

7. Hitchins MP, Stanier P, Preece MA, Moore GE 2001 Silver-Russell syndrome: a dissection of the genetic aetiology and candidate chromosomal regions. J Med Genet 38:810-819

8. National Institutes of Health Consensus Development Conference 1988 Neurofibromatosis. Conference statement. National Institutes of Health Consensus Development Conference. Arch Neurol 45:575-578

9. Jenne DE, Tinschert S, Dorschner MO, Hameister H, Stephens K, Kehrer-Sawatzki H 2003 Complete physical map and gene content of the human NF1 tumor suppressor region in human and mouse. Genes Chromosomes Cancer 37:111-120

10. Wimmer K, Eckart M, Rehder H, Fonatsch C 2000 Illegitimate splicing of the NFI gene in healthy individuals mimics mutation-induced splicing alterations in NF1 patients. Hum Genet 106:311-313

11. Heim RA, Kam-Morgan LN, Binnie CG, Corns DD, Cayouette MC, Farber RA, Aylsworth AS, Silverman LM, Luce MC 1995 Distribution of 13 truncating mutations in the neurofibromatosis 1 gene. Hum Mol Genet 4:975-981

12. Prager S, Wollmann HA, Mergenthaler S, Mavany M, Eggermann K, Ranke MB, Eggermann T 2003 Characterization of genomic variants in CSH1 and GH2, two candidate genes for Silver-Russell syndrome in 17q24-q25. Genet Test 7:259-263

13. Obringer AC, Meadows AT, Zackai EH 1989 The diagnosis of neurofibromatosis-1 in the child under the age of 6 years. Am J Dis Child 143:717-719

14. Stoll C 2002 Difficulties in the diagnosis of neurofibromatosis-1 in children. Am J Med Genet 112:422-426

15. Rosenbaum T, Engelbrecht V, Krölls W, van Dorsten FA, Hoehn-Berlage M, Lenard HG 1999 MRI abnormalities in neurofibromatosis type 1 (NF1). A study of men and mice. Brain Dev 21:268-273

16. Gutmann DH, Aylsworth A, Carey JC, Korf B, Marks J, Pyeritz RE, Rubenstein A, Viskochil D 1997 The diagnostic evaluation and multidisciplinary management of neurofibromatosis 1 and neurofibromatosis 2. JAMA 278:51-57

17. Carmi D, Shohat M, Metzker A, Dickerman Z 1999 Growth, puberty, and endocrine functions in patients with sporadic or familial neurofibromatosis type 1: a longitudinal study. Pediatrics 103:1257-1262

18. Clementi M, Milani S, Mammi I, Boni S, Monciotti C, Tenconi R 1999 Neurofibromatosis type 1 growth charts. Am J Med Genet 87:317-323

19. Szudek J, Birch P, Friedman JM 2000 Growth in North American white children with neurofibromatosis 1 (NF1). J Med Genet 37:933-938

20. Virdis R, Sigorini M, Laiolo A, Lorenzetti E, Street ME, Villani AR, Donadio A, Pisani F, Terzi C, Garavelli L 2000 Neurofibromatosis type 1 and precocious puberty. J Pediatr Endocrinol Metab 13:841-844

21. Baralle D, Mattocks C, Kalidas K, Elmslie F, Whittaker J, Lees M, Ragge N, Patton MA, Winter RM, ffrench-Constant C 2003 Different mutations in the NF1 gene are associated with neurofibromatosis-Noonan syndrome (NFNS). Am J Med Genet 119A: $1-8$

22. Viskochil D, White R, Cawthon R 1993 The neurofibromatosis type 1 gene. Annu Rev Neurosci 16:183-205

23. Cawthon RM, Weiss R, Xu GF, Viskochil D, Culver M, Stevens J, Robertson M, Dunn D, Gesteland R, O'Connell P, et al 1990 A major segment of the neurofibromatosis type 1 gene: cDNA sequence, genomic structure, and point mutations. Cell 62:193-201

24. Wallace MR, Marchuk DA, Andersen LB, Letcher R, Odeh HM, Saulino AM, Fountain JW, Brereton A, Nicholson J, Mitchell AL, et al 1990 Type 1 neurofibromatosis gene: identification of a large transcript disrupted in three NF1 patients. Science 249:181-186

25. Messiaen LM, Callens T, Mortier G, Beysen D, Vandenbroucke I, Van Roy N, Speleman F, Paepe AD 2000 Exhaustive mutation analysis of the NF1 gene allows identification of $95 \%$ of mutations and reveals a high frequency of unusual splicing defects. Hum Mutat 15:541-555 